\title{
Analysis of Assembly Tolerance Based on Assembly Constraint Information Model
}

\author{
Chunxi Li iD and Wenjun Hou \\ Human Computer Interaction Laboratory, Beijing University of Posts and Telecommunications, Beijing 100876, China \\ Correspondence should be addressed to Chunxi Li; lichunxi@bupt.edu.cn
}

Received 18 June 2021; Accepted 19 July 2021; Published 9 August 2021

Academic Editor: Jie Chen

Copyright ( $) 2021$ Chunxi Li and Wenjun Hou. This is an open access article distributed under the Creative Commons Attribution License, which permits unrestricted use, distribution, and reproduction in any medium, provided the original work is properly cited.

\begin{abstract}
Mechanical products are composed of two or more parts. The geometric tolerance and dimensional tolerance of each feature in part will affect the assembly performance of the product, which are accumulated and propagated between assembly fit and parts. In this paper, through the secondary development of CAD software, the B-rep model of parts is obtained. The model information is decomposed and simplified based on geometric features to obtain the key information of parts in the assembly process, simplify the operation, and improve the accuracy. Through a directed graph network, the transmission model of assembly error information based on geometrical and dimensional tolerances (GD\&T) on the surface of parts is established. Combined with the error transfer characteristics of different geometric surfaces and different error sources, guided by the breadth-first search algorithm and the shortest path theory, the search and establishment of a three-dimensional assembly chain are realized. Finally, the three-dimensional chain is simulated by the Monte Carlo method. The calculation results are compared with the error range obtained by the traditional method to prove the effectiveness of the method.
\end{abstract}

\section{Introduction}

Assembly is an integrative process of joining a complete mechanical product by bringing all individual parts together. There is a deviation between the nominal size and the actual size of the product. The manufacturing deviation and fitting deviation are propagated and superimposed in the component through the assembly of parts, affecting the assembly quality and expected function of products. Through tolerance analysis and tolerance stack-up, engineers can predict the final assembly accuracy before machining, so it has become a hot research area of automation [1]. Tolerance stacks are a direct and straightforward method to simulate dimensional deviation on the distance between different assembly features. It usually only includes dimensional tolerance (DT), although modern modification of this method also considers geometric tolerance [2]. However, under the actual manufacturing conditions, the propagation of geometric, dimensional tolerances (GD\&T), the gap between mating parts, and the assembly sequence of parts will cause some mating features to deviate from their nominal positions, thus affecting the assembly accuracy [3].

In recent years, scholars have established general tolerance analysis methods [4-8], which can obtain the assembly accuracy of mechanical products to a certain extent. However, few researchers consider the influence of the assembly sequence on assembly accuracy. Assembly sequence planning and assembly accuracy calculation depend on the assembly information model. To analyze the influence of the assembly sequence in the calculation of assembly accuracy, we should establish an appropriate model that can carry out assembly sequence planning and assembly accuracy calculation. The appropriate model needs to include the dimension and tolerance information of parts and the assembly relationship information between parts and components for assembly [4]. GD\&T and assembly relationship information are added into parts' geometric features and propagated through parts' direct geometric contact. 
To model assembly information, several problems need to be further studied. First of all, only some features influence the assembly process, so we are only aiming the key features to do the subsequent calculation. Secondly, the surface of parts determines geometric constraints between parts and geometric feasibility of the assembly. Nongeometric constraints have an influence on assembly efficiency. It is necessary to combine the nongeometric constraints that affect the assembly sequence to optimize the feasible geometric sequence. Different tolerances on different types of assembly surfaces will have different effects on assembly accuracy, so it is necessary to establish an assembly error transfer model to obtain error transfer and superposition in parts.

This paper presents a method to obtain the assembly dimension relation model. The key features of part obtained by simplifying the part and determining the influence area of error according to the different contact surfaces. Then, the complete assembly dimension relationship model is formed by combining the assembly error. Based on the model, the three-dimension chain between the corresponding features of two different parts of the assembly is searched by the graph search algorithm and solving the assembly parts' assembly error. The basic process is as follows: (1) the hierarchical assembly information model can establish the decomposition of the geometric feature of the part represented by the B-rep model. (2) The assembly information model carries out assembly sequence planning. (3) On the premise of obtaining the optimal assembly sequence under multiple constraints, the three-dimensional chain of parts in an assembly accuracy propagation model is searched. (4) The assembly accuracy of related parts is calculated based on obtaining the dimension chain.

\section{Literature Review}

2.1. Assembly Constraint Model. The optimal assembly sequence has an important influence on assembly time and cost $[9,10]$. For generating the valid assembly sequences, the engineers must be pointed out the assembly precedence between parts. However, it is challenging and time-consuming to find the optimal assembly sequence due to many feasible assembly sequences. Assembly sequence planning (ASP) is typical of the discrete optimization problem in mathematics. In the early 1960s, researchers developed at least one optimal feasible assembly sequence for a product [11]. Then, through the assembly constraints between parts, the feasible assembly sequence can be found using the graphical method [12]. Although these traditional methods provide a correct and complete solution for the assembly configuration of some parts counting, they were timeconsuming and required many calculations [13]. Also, the methods were semi-automated, which required skilled users to oversee the decision-making process.

In 1988, graphic representation methods such as connection graph, interference graph, and assembly constraint graph were proposed to represent assembly trajectory constraints and were used to generate assembly priority relationships [14]. de Mello and Sanderson introduced all feasible assembly sequences of the product with the and/or graph [15]. In the graph, the node is an assembly subpart, the root node is a complete part, the leaf is a separate part, and the arc corresponds to a feasible assembly operation, linking each node to all combinations in which it can be split [16]. Wolter, on the other hand, used a partial assembly tree to express the assembly. Its nodes correspond to subassemblies in the assembly process. The leaf node is a single part, while the root node is the final mechanical product [17]. Besides, there is an assembly directed graph $[9,18]$, whose nodes correspond to the assembly of parts in the assembly and whose arcs correspond to feasible assembly operations [19].

A researcher prefers extracting assembly sequence constraints from Computer-Aided Design (CAD) software to minimize human intervention. Some researchers have developed automatic extraction methods to retrieve assembly connection data, interference data, and assembly stability information through CAD interfaces [20, 21]. However, this method requires manual operations, which rely on engineers' experience, so it is not the optimal solution as human input errors are a significant factor [22]. The existing methods cannot automatically establish the appropriate assembly constraint information model. In this paper, the surface included in the part is divided to obtain the geometric structure and the topological relationship between the geometric structures. The introduction of model simplification reduces the number of nodes in the model, improves operation efficiency, and results in faster and more accurate results. The key features that affect the assembly accuracy are combined with the nongeometric information to form a complete assembly information model.

2.2. Error Transformation Model for Assembly. The design and calculation of dimensions and tolerances are difficult problems in the industry. Scholars have carried out much research on the mathematical model expression of assembly tolerance to solve this problem. Typical tolerance models include topologically and technologically related surface (TTRS) [7, 23], polychromatic set model [24], small-displacement sensor (SDT) [25], tolerance map (T-map) [5], and degree of freedom model [26].

As a widely used model, Clément et al. [27] proposed the TTRS model on seven-element surfaces, and twenty-eight surface relationships determine the tolerance. Davidson and Shah [28] established the T-map tolerance model of standard plane features. On this basis, Xiao and Zhu [29] combined with T-map and ASME standard, took the intersection axis as the research object, established the spatial domain of assembly feature deviation fluctuation, and improved the accuracy of the transformed tolerance. Solving the problem that the machining datum does not coincide with the design datum in the machining process, the tolerance graph is another model for calculating tolerance in the $n$-dimensional Euclidean space, mapping the geometric features of parts to the point space model. Ke et al. [30] established an M-map to realize the transfer of the datum plane and realized the adjustment and optimization of machining tolerance under different constraint conditions. 
The stack-up of assembly errors is another problem in the process of assembly accuracy calculation. Liu et al. [31] divided the assembly errors into three categories: geometric position deviation, geometric shape deviation, and assembly position deviation, and then, they established the assembly directed graph to express the transmission process of assembly accuracy. Boccaletti et al. [32] divided a complex network into different modules based on complex network and dynamic cluster coefficient analysis, which provides a new idea of error tracing. Based on the above principles [33], Zhu et al. [34] established the deviation transfer network model of the mechanical assembly process, identified the key assembly surface, and realized the error traceability. The existing methods are limited to the transfer direction of assembly error. In addition, few researchers regard the assembly sequence as one of the constraints of assembly accuracy. However, different assembly sequences will lead to different contact sequences between parts and different error transmission paths. In this paper, in the same model, the optimal assembly sequence of parts is deduced, and the assembly accuracy of parts is obtained under this sequence.

\subsection{Problem Definition and Objectives of Present Research.} Every feature in a component is subjected to variation between design positions and is strictly applied with some tolerances. When there are multiple components in a mechanical product, the variations will accumulate and propagate, affecting the functional performance of parts. The sources of these changes can be divided into three categories:

(1) The size change or tolerance superposition of a single part

(2) The change of geometric features

(3) The change of parts and the change of transmission between parts in the assembly process

In previous studies, the second and third types of deviations are not considered in evaluating fit accuracy, which usually regards as null according to the translation or selection changes added in the assembly process.

The variation of geometric features can significantly affect tolerance superposition, depending on the type of translation and rotation variation added during assembly. In the process of assembly accuracy simulation, considering the assembly sequence of mechanical products and the matching accuracy between parts, the assembly accuracy obtained is more realistic than the simple dimensional accuracy. The propagation direction of different error sources on different surfaces is also different. In searching the dimension chain, it is necessary to determine whether each error has a component in the calculation direction of assembly accuracy and then ignore the interaction of multiple errors. So, the expected accuracy of precision assembly can be calculated, and the service life of parts can be improved.

This paper aims to apply geometric tolerance and dimensional tolerance, surface tolerance, assembly error, and assembly sequence of parts to the calculation of assembly accuracy. Therefore, it is necessary to establish a model containing all the assembly information of the parts. The influence of geometric constraints between parts on the feasible motion range of parts is analyzed. It is important to determine the feasible transfer direction of assembly deviation of different kinds of error sources on different surfaces. In establishing the model, the key features of the parts participating in the assembly process are obtained through simplification and planning the assembly sequence of the products. After obtaining the contact sequence between parts, it is necessary to analyze the influence of GD\&T deviation on assembly accuracy. We propose a breadth-first algorithm combined with the assembly sequence to search the $3 \mathrm{D}$ chain of the required assembly size in the assembly error transfer model to solve this problem. Finally, the assembly accuracy of the product is obtained by the Monte Carlo method. The shaft and its related parts of the two-stage reducer are an example. Analyzing is carried out in order to understand the proposed method.

\section{Assembly Information Model}

The assembly constraint relation model expresses constraint and mating relations between parts. Assembly tolerances can be used as attributes of assembly models and are attached to them. This paper builds a hierarchical representation model whose structure is shown in Figure 1. The representation model includes five levels: assembly level, part level, assembly feature level, surface level, and assembly tolerance level.

To meet the design requirements, CAD models usually contain accurate geometric information. There are some structures in part, such as chamfering, fillet, or tool retracting groove, which will increase the number of nodes in the assembly information model and increase calculation difficulty. Therefore, it is necessary to calculate the key features of parts to improve operational efficiency. In this paper, by simplifying the model extracted from CAD, the key features with assembly constraints are obtained. If a simplified basic unit is a surface, the part may not be a complete entity. To avoid this problem, take the feature as the minimum simplified element. Because different engineers understand that the CAD modeling process is different, using feature trees directly in CAD software cannot get the simplest simplified results. Therefore, through the secondary development of the CAD system, the digital model of part B-rep representation is obtained. This method generates a feature-based model from the B-rep model by applying the volume decomposition method. The assembly information model of mechanical products is constructed hierarchically to store the assembly constraints and the nongeometric information related to the parts.

3.1. B-Rep Model. Obtaining the geometric constraint information between parts, through the contact surface between parts, we can obtain the geometric structure of parts, including contact surface and noncontact surface. The noncontact surface ensures the transmission of geometric constraints on the part, while the contact surface ensures the transmission of geometric constraints between the part. The 


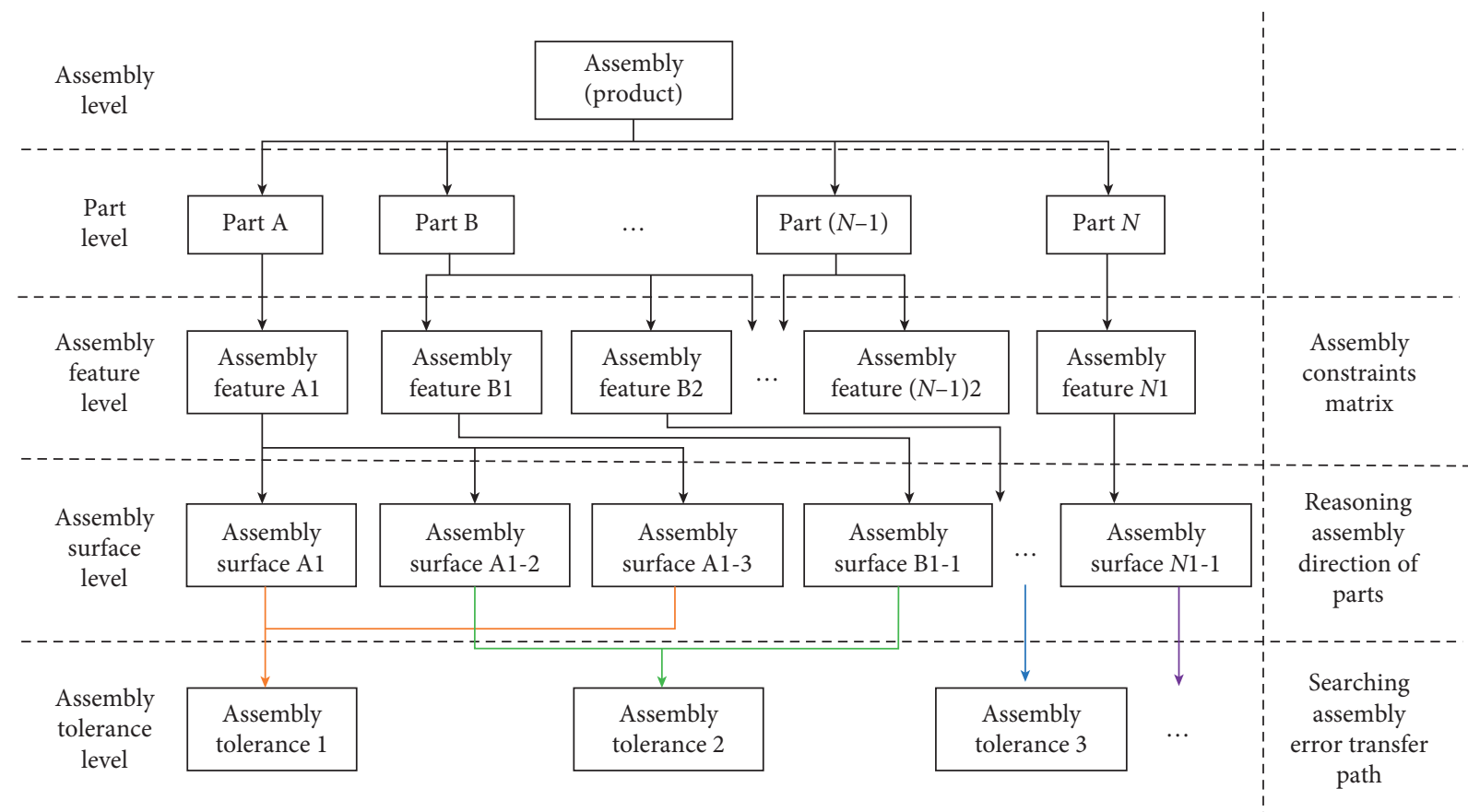

FIgURE 1: Assembly information model of the product.

B-rep model established the model of parts' contact relationship through the secondary development of CAD. The feature tree of the part is obtained through the decomposition of the volume feature.

The assembly information model can represent constraint information at each level and indicate the reasoning relation matrices built at different levels. Boundary representation is a common geometric description method in CAD. The B-rep model retains the information of points, lines, and surfaces in the CAD model, which helps calculate the geometric features of parts. In the B-rep model, as shown in Figure 2, the type of part surface is obtained, and the concave-convex of the edge formed by the intersection of two surfaces is judged by the angle between the normal vectors of two surfaces.

3.2. Geometric Feature Decomposition. The mechanical product can be seen as a complex geometry formed by many simple subfeatures and their Boolean operation. When applying additive features, convex inner loops are often generated at feature intersections. So, the convex inner loops can be the sign of additive features. Koo and Lee [35] proposed the wrap-around operation to decompose parts. The algorithm for the wrap-around operation on a given shape $S$ is described as follows:

(1) Find and mark the convex inner loops $\left\{L_{i}\right\}$ of $S$ (Figure 3(a)).

(2) Separate every faces $\left\{F_{j}\right\}$ (Figure $3(b)$ ).

(3) Find $\left\{L_{i}\right\}$ in $\left\{F_{j}\right\}$ (Figure 3(b)).

(4) Remove $\left\{L_{i}\right\}$ and add new surface according to $\left\{L_{i}\right\}$ (Figure 3(c)).

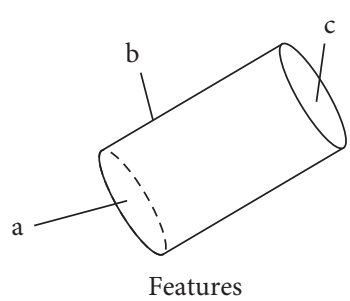

(a)

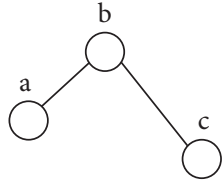

B-rep model

(b)
Figure 2: B-rep model for parts.

(5) Sew all $\left\{F_{j}\right\}$ and add corresponding plane (Figure 3(d)).

(6) Unite $T$ and $W$ with a regularized Boolean union (Figure 3(d)).

(7) If $T$ has convex inner loops, apply the wrap-around operation again to $T_{1}$ and obtain $T_{2}$. Repeat the wraparound operation until convex inner loops do not exist.

3.3. Assembly Constraints Model. Most studies on the simplification of feature-based 3D CAD models involve the following two steps: feature rearrangement according to importance and removal of features with low importance until the termination condition. In this study, through the effective volume of features and the contact features between parts, the parts are simplified on the premise of maintaining the connectivity of the model using a feature adjacency graph.

A feature tree is generated by decomposing the part and reconstructing the simple subfeatures. Different deletion 


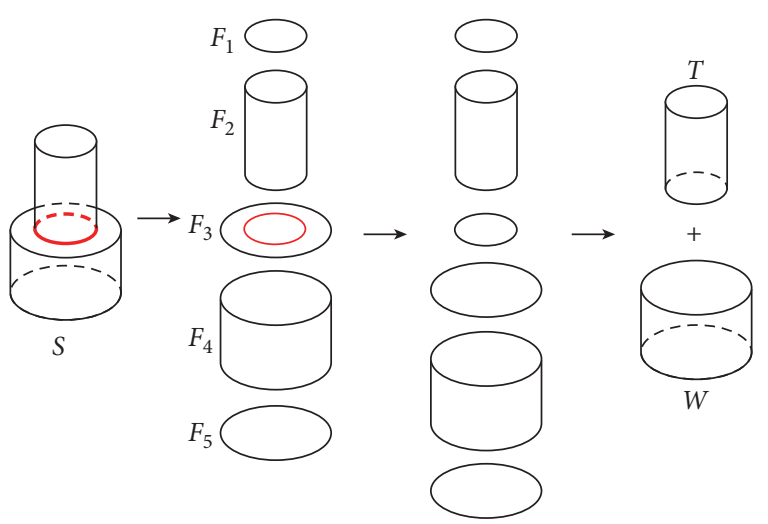

Figure 3: Concept of volume decomposition.

processes lead to different results, so it is necessary to determine a reasonable deletion order to ensure the integrity of the constraint information of the final part. Kim and Mun [36] and Kang et al. [37] proposed several recombination strategies. This study evaluates the importance of subfeatures according to the following criteria. The main results are as follows:

(1) The importance of additive features is higher than that of subtractive features.

(2) Larger features are more important than smaller ones.

(3) Features need to be retained in contact with other parts.

(4) The first adjacent feature near the port is more important than the other features. We use the following formula to measure the importance of features:

$$
f=n \times\left(C_{1}+C_{2}+C_{3}\right) .
$$

If it is a port feature, it cannot be deleted and retained. At this time, $n=1$, making $f>0$. Otherwise, $n=-1$ making $f<0$. $C_{1}$ is the volume ratio of the feature to the largest feature in the part, which is a number greater than 0 and less than 1 . If it is an additive feature, $C_{2}=1$; otherwise, it is 0 . Suppose that it is the first feature near the port, $C_{3}=1$. Otherwise, it is 0 . The results are shown in Figure 4.

As shown in Figure 5, all the nodes are connected, and all the nodes in the graph are ordinary nodes. When deleting node 4 , node 2 will become the cut node, while node 3 , node 5 , and node 1 remain unchanged. After the deletion of node 3 on the above condition, all the nodes in the graph become ordinary nodes. Thus, the graph nodes can switch between cut nodes and ordinary nodes in the simplification process. Furthermore, the set of cut nodes need to reevaluated when the graph changes.

During the reduction of nodes on the diagram in the graph above, the nature of the retained node as a cut node also changes. Therefore, it is necessary to determine whether the node in the graph is a cut node after deleting a feature. When the node to be deleted is a cut point in the geometric contact diagram, skip the feature. Then, the part model is simplified according to the order.
The assembly constraints graph is highlighting the features from the same part in the same color. The node code is a 4-digit string. The first two digits are the $I D$ of the part, and the last two digits are the ID of the face in part, as shown in Figure 6(d).

3.4. Assembly Variation Model. A complex component is considered to have a high number of parts and a large degree of connectivity between subfeatures or parts. Most of the existing assembly accuracy analysis methods simplify the machining error of the part surface to the translation and rotation of the ideal geometric surface along with its theoretical position. This simplification ignores the influence of surface morphology on the position of parts, thus affecting the accuracy of precision analysis results.

The assembly tolerances mentioned in this paper include dimensional tolerances, shape tolerances, and position tolerances. Form tolerances and position tolerances are collectively referred to as geometric tolerances. The error of the part can be regarded as an ideal surface, which is translated and rotated under the limitation of orientation and positioning error and then superimposed with the deformation caused by shape tolerance. Therefore, the small-displacement torsor (SDT) represents the orientation and positioning error of the part, and then, the shape error is expressed in the form of the point cloud. The superposition of the two is the final assembly error .

\section{(a) Positioning Tolerance Modeling.}

When geometric features are constrained by location tolerance and orientation tolerance, location and orientation errors can be expressed by SDT, which include a set of translation components along the $x$ axis, $y$-axis, and $z$-axis $([\mathbf{u}, \mathbf{v}, \mathbf{w}])$ and a set of rotation components along the $x$-axis, $y$-axis, and $z$-axis $([\alpha$, $\beta, \gamma])$. The SDT representation of common geometric features and their value range is shown in Table 1. In Table $1, L$ and $W$ represent the length and width of the rectangular plane, $\varphi d$ and $\varphi D$ represent the outer diameter of the circle, and $H$ and $h$ represent the height of the cylinder and cone. On this basis [38], the geometric surface with positioning/orientation errors can be obtained by translating and rotating the geometric surface in the ideal position, and the given SDT parameters determine the values of translation and rotation.

(b) Form Tolerance Modeling.

Similar to positioning or orientation tolerance, form tolerance also limits the variation range of geometric features. The rectangular plane is taken as an example; its form tolerance region is also the area between two parallel planes, as shown in Figure 7. The nonideal surface generated by form error modeling still needs to meet the constraints of form tolerance. Form error can be modeled by point cloud simulation. 

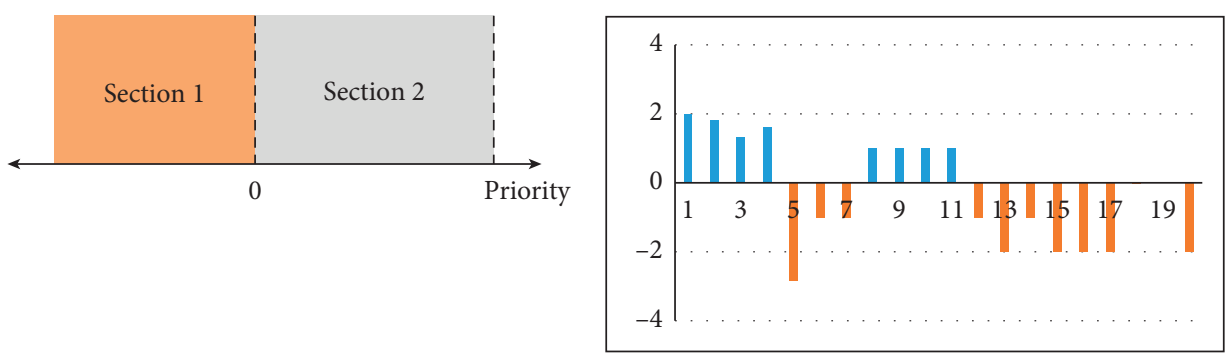

(a)

(b)

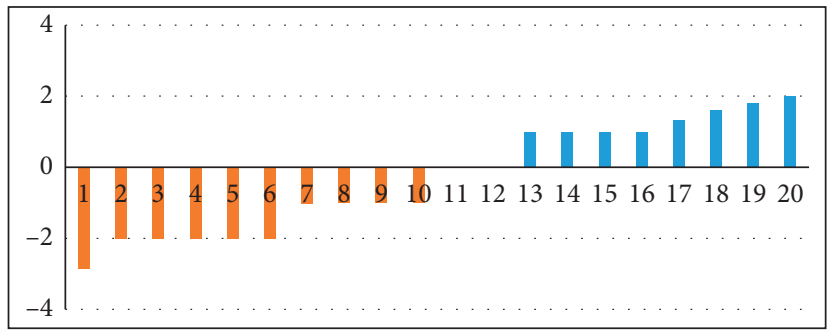

(c)

FIGURE 4: Geometric features are rearranged according to their importance. (a) Two sections according to feature importance. (b) Importance of the feature. (c) Rearrange the features according to their importance.

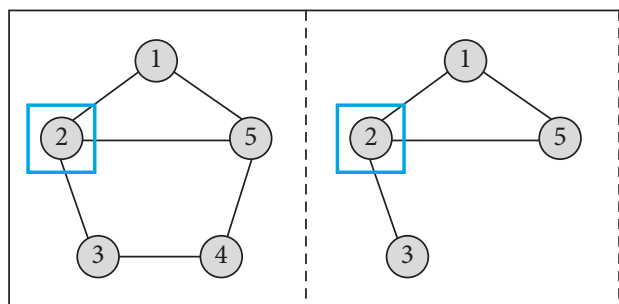

(a)

(b)

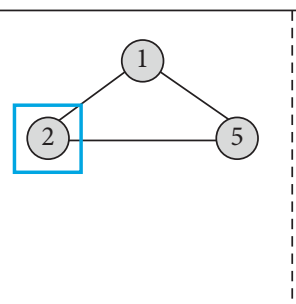

(c)

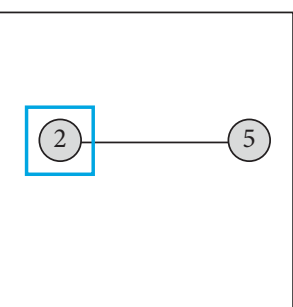

(d)

Figure 5: The change of cut points in the process of node deletion in the undirected graph.

The nonideal surface model is generated by superimposing a randomly generated geometric surface with form error on the geometric surface with location and orientation error based on the modeling of location, orientation error, and form error, as shown in Figure 8. Form tolerance and position tolerance are independent of each other, and they constrain the surface of parts together. It should be noted that, in the process of using numerical simulation, in some cases, the plane will exceed the constraint range of tolerance, so manual correction is needed.

\section{Assembly Sequence Planning}

The essence of part assembly is to impose constraints on geometric elements to adjust the position and the rotation of the part model. The geometric elements involved in the constraints between parts include various geometric surfaces, such as planes, cylinders, and cones. This paper focuses on the geometric surfaces with contact relationships between two different parts.
4.1. Geometrically Feasible Assembly Sequence. In 3D space, the assembly constraints of different kinds of geometric surfaces are transformed into the remaining feasible motion direction of the part. The unit spherical coordinates are stored in the form of a set, as shown in Figure 9.

Table 2 shows the constraints of the geometric contact surface in part on the feasible direction of movement and the assembly direction. When there are constraints between the planes, the part can move in the opposite hemisphere of the plane normal vector, and the assembly direction is the normal direction of the plane; when there are cylindrical constraints, the part can move in the positive and negative direction of the vector, and the assembly direction is also the positive and negative direction of the vector; when there are conical constraints, the part can move in the opposite direction of the vector, and the assembly direction is the component of the vector.

The potential assembly sequence for $N$ parts in a mechanical product is $N$ !. Geometric constraints between parts result in a reduction in the number of parts. The method of 


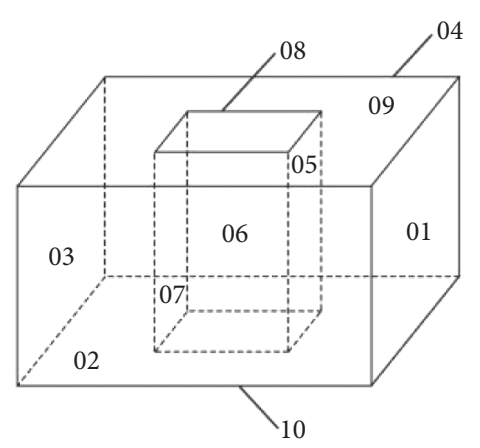

(a)

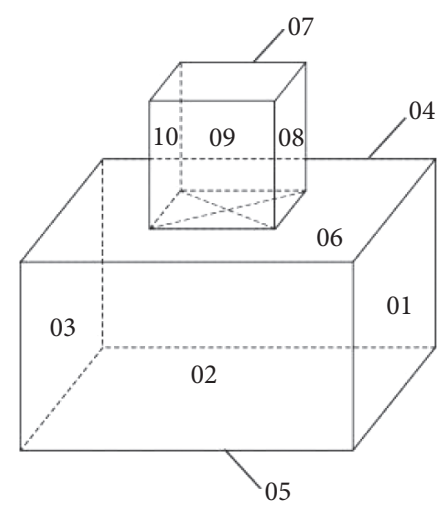

(b)

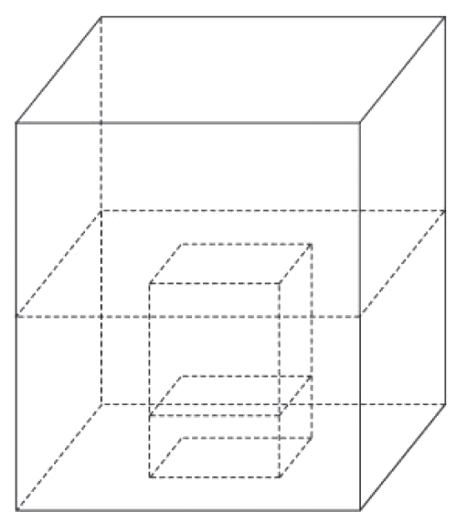

(c)

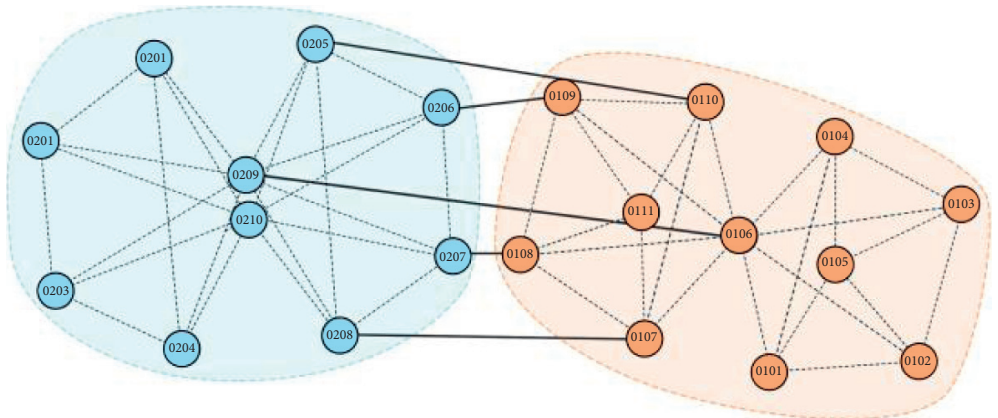

(d)

Figure 6: Contact relationship of parts based on surface representation. (a) Part 1 CAD model. (b) Part 2 CAD model. (c) Fitting model of parts. (d) Feature representation of part fitting model.

TABLE 1: Analysis of the tolerance zone in common geometric features.

Deviation vector

obtaining the assembly sequence is to inverse the disassembly sequence. In this paper, a geometrically feasible assembly sequence for all parts is obtained by continuously removing the parts from the product.

An undirected graph of assembly constraints of mechanical products is established through the port features of parts and the B-rep model of assembly. The node represents the contact surface between parts and records the type and direction of the surface. The assembly sequence is represented as a string, so the sequence [1 3456789101516212 11 13] is represented as " $01,03,04,05,06,07,08,09,10,15$, $16,02,12,11,13$," and each part number is represented by two digits. Part 2 has $I D$ number 02 , and part 13 has $I D$ number 13.

Parts with the not empty feasible moving area can be disassembled when constraints are received. Then, surface nodes and connections between surface nodes and link edges can be deleted, which contact other parts. Record the removed parts, update the assembly constraint relationship model, and then, obtain the parts with no empty feasible moving area again. When there are several removable parts, store them separately to form several strings representing the disassembly sequence. Repeat this process until all parts are completely removed. Invert the obtained disassembly 


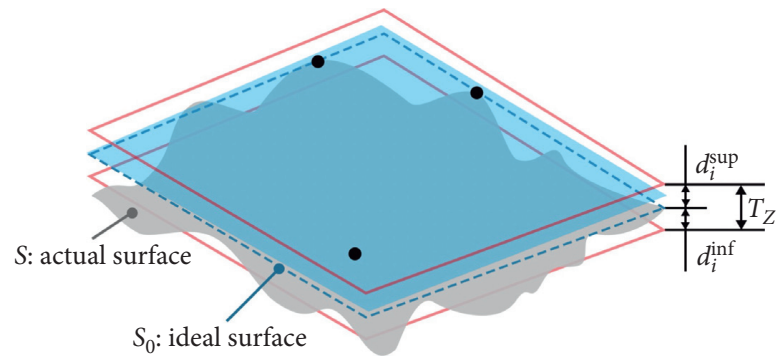

FiguRe 7: Form error of the plane.

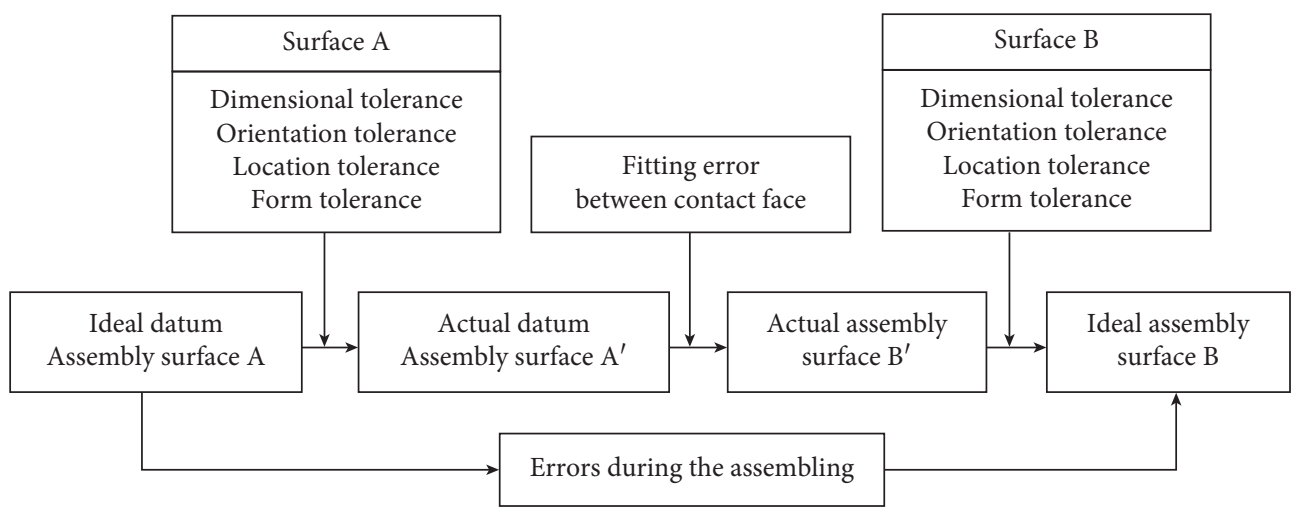

FiguRE 8: Error transfer between two surfaces.

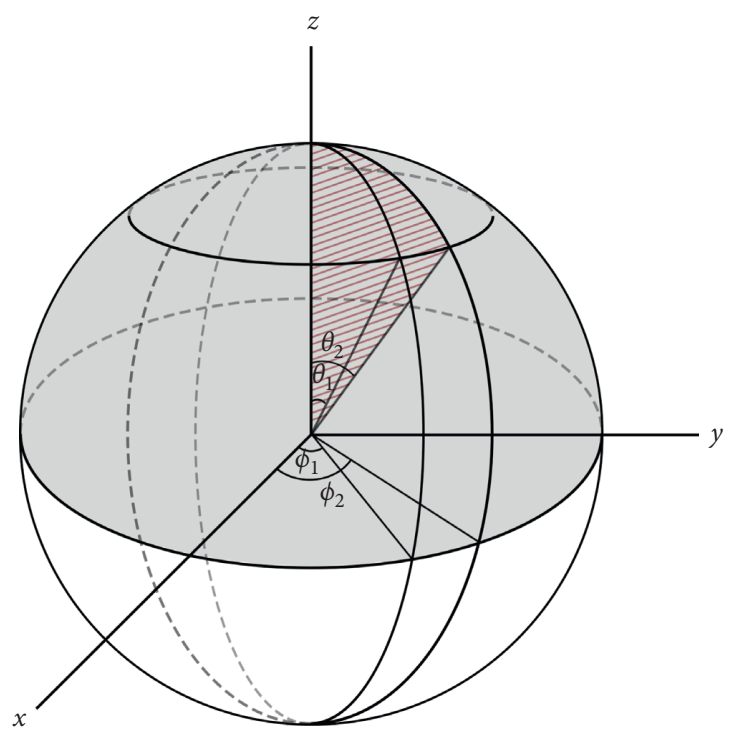

FIGURE 9: Feasible assembly region represented by spherical coordinates.

sequence to define the geometrically feasible assembly sequence of a part.

For example, there are two pairs of surface contact in model $\mathrm{A}$ and model $\mathrm{B}$. They are marked as $x_{1}, y_{1}, x_{2}$, and $y_{2}$, and the assembly direction constraint of the part between $x_{1}$ and $x_{2}$ is shown in Figure 10(a), and the constraint of the part between $y_{1}$ and $y_{2}$ is shown in Figure 10(b). The feasible assembly domain of the two parts is shown in Figure 10(c). The details are shown in Figure 10.

4.2. Assembly Sequence Optimization. Before optimizing the assembly sequence, it is necessary to determine the fitness function, which describes the difficulty and complexity of 
TABLE 2: Constraints of the surface on the feasible assembly region of parts.

\begin{tabular}{|c|c|c|c|c|c|c|}
\hline $\begin{array}{l}\text { Connect } \\
\text { relationship }\end{array}$ & $\begin{array}{l}\text { Assembly } \\
\text { direction }\end{array}$ & $\begin{array}{c}\text { Disassembly } \\
\text { direction }\end{array}$ & $\begin{array}{l}\text { Assembly zenith } \\
\text { angle }\end{array}$ & $\begin{array}{c}\text { Assembly } \\
\text { azimuth angle }\end{array}$ & $\begin{array}{l}\text { Disassembly } \\
\text { zenith angle }\end{array}$ & $\begin{array}{l}\text { Disassembly } \\
\text { azimuth angle }\end{array}$ \\
\hline & & & $\theta=[(\pi / 2), \pi]$ & $\phi=[0,2 \pi]$ & $\theta=[0,(\pi / 2)]$ & $\phi=[0,2 \pi]$ \\
\hline & & & $\theta=[0,0]$ & $\phi=[0,2 \pi]$ & $\theta=[0,0]$ & $\phi=[0,2 \pi]$ \\
\hline & & & $\theta=[\pi, \pi]$ & & $\theta=[\pi, \pi]$ & \\
\hline & & & $\theta=[0,0]$ & $\phi=[0,2 \pi]$ & $\theta=[\pi, \pi]$ & $\phi=[0,2 \pi]$ \\
\hline
\end{tabular}

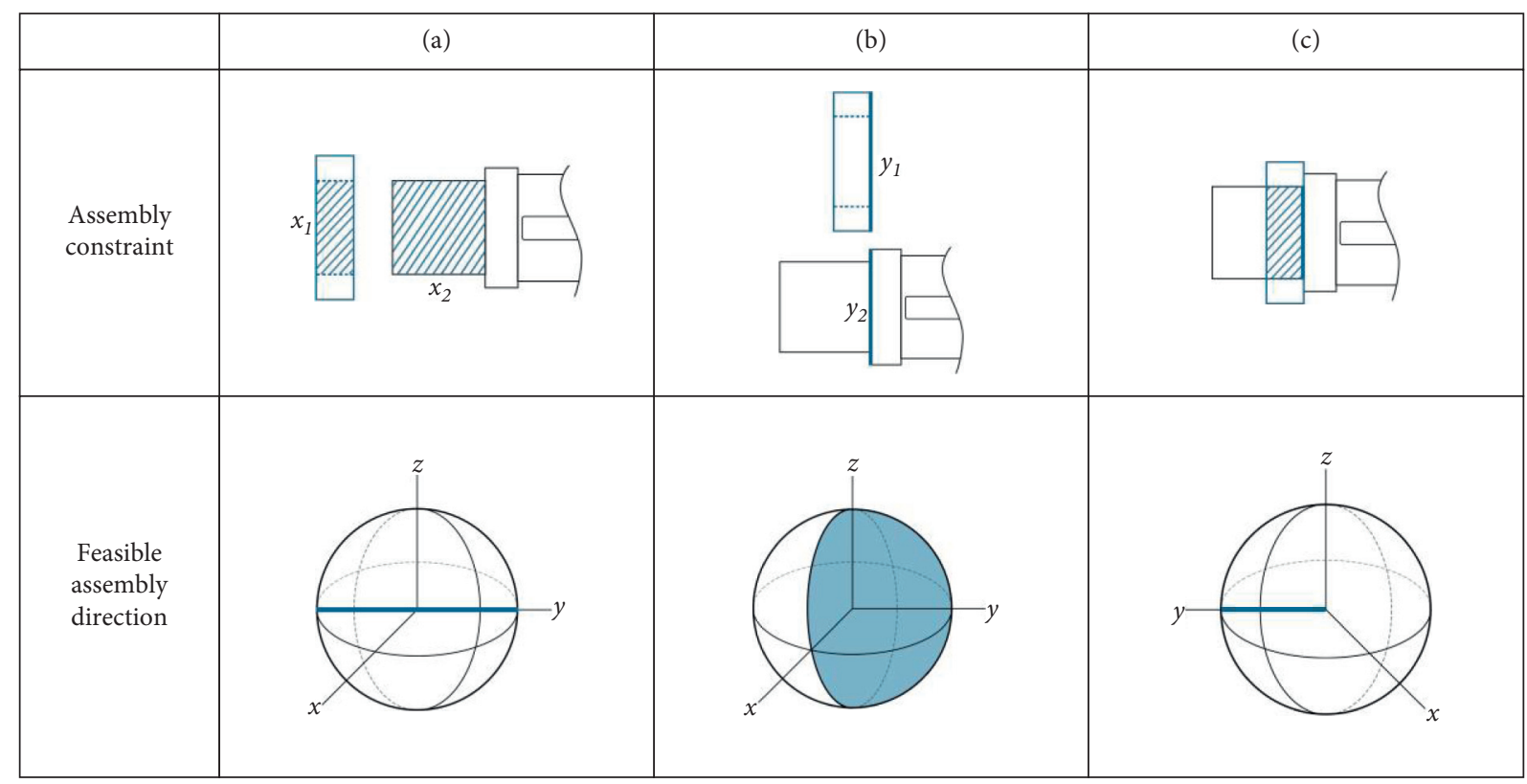

FIGURE 10: Determination of the assembly region between parts.

assembly sequence operation. The fitness function includes the following:

(1) The number of tools changes in the assembly process

(2) The number of times the assembly direction changes in the assembly process

(3) The fitness difficulty between parts

The optimization function used in this paper is shown in formula (2), and the calculation method of variables is shown in Table 3.
$F_{n}$ represents the assembly process factors, including the change times of assembly tools $R_{1}$, the difficulty of assembly operation $R_{2}$, and the change times of assembly direction $R_{3}$. Xie and Zhong used the analytic hierarchy process to evaluate the assembly sequence, in which the weights of various constraints were obtained by consulting experts using the sadi scale. This paper referred to the relevant conclusions [39]. The value of weight factors was set as $\omega_{1}=0.2, \omega_{2}=0.3$, and $\omega_{3}=0.5$. Then, using the fitness function to select the geometric feasible assembly sequence to get the optimal solution under the constraint: 


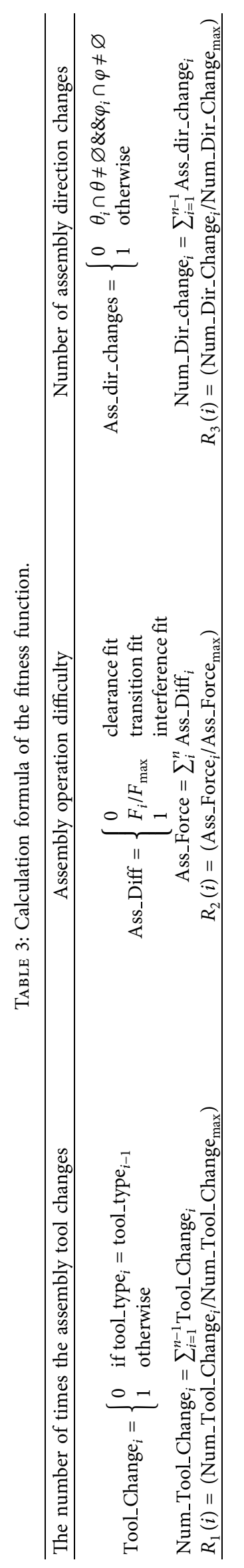




$$
F_{n}=\omega_{1} R_{1}+\omega_{2} R_{2}+\omega_{3} R_{3} .
$$

\section{3D Dimension Chain Search Based on the Breadth-First Algorithm}

According to the assembly error model established in the previous paper, the assembly error of parts can be obtained by calculating the three-dimensional dimension chain of mechanical products. According to the assembly sequence, we need to obtain the error transfer path in each part of the product and the error transfer relationship between parts and gradually obtain the three-dimensional chain of this precision. When the number of nodes increases, the accumulated error on the path also increases, so transforming the assembly accuracy calculation problem can be transformed into a shortest path problem.

Based on the model network established in the previous paper, a directed graph $G$ is constructed, in which the faces are mapped to the node $\mathrm{n}$ of $\mathrm{G}$ and the transfer relationship of assembly errors between faces is mapped to the edge $e$ of $G$. For the edge $e$, if the precision level between two sides is high, the distance $W(e)$ of edge $e$ is smaller. If the precision level between two sides is low, the distance $W(e)$ of edge $e$ is larger. If there is no component of the error precision between two sides in the band direction, then $W(e)$ is a large number $M$. Let $d\left(v_{i}, v_{j}\right)$ be the set of paths with $u$ and $v$ as the endpoints in $G$ and $W\left(d\left(v_{i}, v_{j}\right)\right)$ denote the sum of the distances on the top of the path $d\left(v_{i}, v_{j}\right)$. The three-dimensional dimension chain search problem can be expressed as finding a path $P_{0}\left(v_{i}, v_{j}\right)$ in $d\left(v_{i}, v_{j}\right)$ in $G$ such that

$$
W\left(P_{0}\left(v_{i}, v_{j}\right)\right)=\min \left(W\left(p\left(u_{i}, v_{j}\right)\right) \in d\left(u_{i}, v_{j}\right)\right) .
$$

Typical shortest path algorithms include Dijkstra algorithm, Floyd algorithm, Bellman-Ford algorithm, and SPFA algorithm. In this paper, we get the shortest path when the starting vertex and the ending vertex are known. Therefore, we are choosing the Floyd algorithm as the search algorithm for the $3 \mathrm{D}$ dimension chain.

The Floyd algorithm thought is that the shortest path from any node $v_{i}$ to any node $v_{j}$ is no more than two possibilities, one is directly from node $v_{i}$ to node $v_{j}$ and the other is from $v_{i}$ through several nodes to $v_{j}$. Suppose that distance between node $u$ and node $v$, which is expressed as $\operatorname{dist}\left(v_{i}, v_{j}\right)$, is the shortest path distance from node $v_{i}$ to node $v_{j}$ for each node $k$, and check whether $\operatorname{dist}\left(v_{i}, k\right)+\operatorname{dist}\left(k, v_{j}\right)<\operatorname{dist}\left(v_{i}, v_{j}\right)$ holds. If it is true, it is proved that the path from node $v_{i}$ to node $k$ and then to $v_{j}$ is shorter than the path from $u$ to $v$. After traversing all nodes $k$, dist $\left(v_{i}, v_{j}\right)$ records the shortest path distance from $v_{i}$ to $v_{j}$.

The error transfer graph $G=\{V, E\}$ of each part and the assembly sequence $\mathbf{s}=[a(i)]_{n}$ under multiple constraints are calculated. The weight adjacency matrix $\tau=[a(i, j)]_{n \times n}$ is constructed, and a subsequent node matrix path $\mathbf{p}=[a(i, j)]_{n \times n}$ is introduced to record the shortest path between two points. The specific algorithm steps are as follows:
(1) Determine the transfer direction of the error. According to the start and end surfaces of assembly error, spherical coordinates are recording the error transfer direction.

(2) This can be obtained between the contact surfaces and the contact order of the parts by the determined assembly sequence. Judge whether there is a component in the direction of error transmission. The error transfer path between parts, the participating parts, and the start and end surfaces of the participating parts is obtained

(3) For each part, the weighted error transfer digraph is established from any path. The distance between all the two points is the weight of the edge. If there is no edge connection between the two points, the weight is infinite. The graph weight adjacency matrix and node matrix path are constructed.

(4) Use the Floyd algorithm to find intermediate node $k$. If there is a vertex $k$, update the result.

(5) Repeat the above step (2)-step (4) until all nodes are traversed until the end. The shortest path formed is the three-dimensional dimension chain of parts.

(6) Through the dimension chain of parts and the fitting error between parts, the three-dimensional dimension chain of the error to be solved is formed.

\section{Assembly Accuracy Calculation}

After obtaining the three-dimensional dimension chain of the product, the assembly accuracy of the product can be calculated, including the error transfer and numerical calculation.

6.1. Error Transfer of Homogeneous Transformation Matrix. Based on the coordinate transformation theory of robot kinematics, a $4 \times 4$ homogeneous transformation matrix is used to express the tolerance model transmit [40]:

$$
\mathbf{M}=\left[\begin{array}{cccc}
1 & -\gamma & \beta & u \\
\gamma & 1 & -\alpha & v \\
-\beta & \alpha & 1 & w \\
0 & 0 & 0 & 1
\end{array}\right] .
$$

Then, the transformation relation of the coordinate position of $P$ point from $S 2$ to $S 1$ is as follows:

$$
\mathbf{p}_{s 2}=\left[\begin{array}{c}
X_{s 2} \\
Y_{s 2} \\
Z_{s 2}
\end{array}\right]=\mathbf{T} \bullet\left[\begin{array}{c}
X_{s 1} \\
Y_{s 1} \\
Z_{s 1}
\end{array}\right]=\mathbf{T} \bullet \mathbf{P}_{s 1} .
$$

As shown in Figure 11, the nominal coordinate system and the actual coordinate system of the geometric features of the mating surface are established at the mating surface between parts. They represent mapping of the measuring point $P$ from one space coordinate system to another space coordinate system by the homogeneous coordinate transformation matrix. 

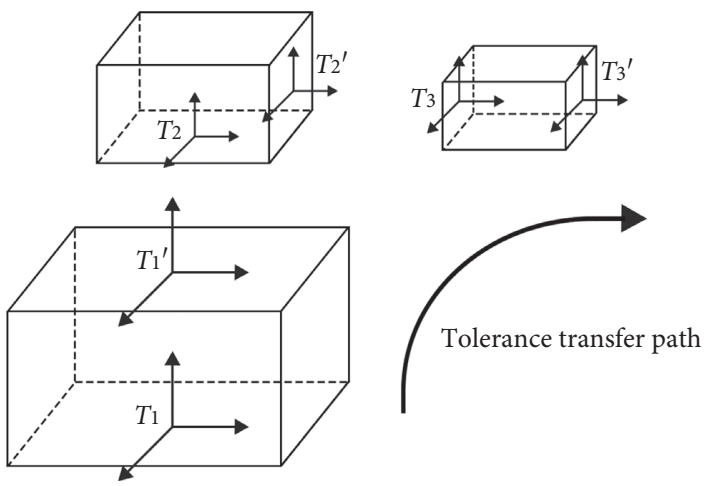

Figure 11: Tolerance transfer path in the product.

The transformation of any mating surface from the nominal feature coordinate system to the actual feature coordinate system will affect the spatial attitude of subsequent parts in the global coordinate system. For the measurement point $P$, its spatial position and pose in the global coordinate system will be affected by the surface changes of each link in the dimensional chain. It is mathematically described as a cross product of the series transformation matrices of all adjacent coordinate systems on the transfer path:

$$
\mathbf{P}_{s 3}=\left(\mathbf{T}_{11} \bullet \mathbf{T}_{12} \bullet \mathbf{T}_{22} \bullet \mathbf{T}_{23} \bullet \mathbf{T}_{33}\right) \mathbf{P}_{s 1} .
$$

Among them, $\mathbf{T}_{11}, \mathbf{T}_{22}$, and $\mathbf{T}_{33}$ represent the coordinate transformation matrix of the actual mating surface relative to the nominal mating surface between parts $\mathbf{P}_{1}, \mathbf{P}_{2}$, and $\mathbf{P}_{3}$. $\mathbf{T}_{12}$ and $\mathbf{T}_{23}$ represent the relative transformation matrix of nominal coordinates between parts $\mathbf{P}_{1}$ to $\mathbf{P}_{2}$ and $\mathbf{P}_{2}$ to $\mathbf{P}_{3}$.

6.2. Calculation Accuracy Based on Monte Carlo Method. In the process of calculating assembly accuracy, the process of tolerance superposition optimization is extremely complex. An optimization strategy combined with the Monte Carlo method has been proposed to solve this problem. According to the distribution law of the uncertain values in the error domain, the specific size of each assembly is randomly selected, and the error domain of the final assembly error is obtained by multiple stack calculation.

In the three-dimensional dimension chain of error transfer, for the numerical simulation of the errors contained in the nodes, the manufacturing errors usually conform to the normal distribution. Through the statistical simulation, the approximate solution of the assembly error is obtained. After determining the three-dimensional dimension chain of assembly error transfer, the number of simulation $N$ needs to be determined, and the distribution function of error sources determines each error source in the chain, and random sampling is carried out within the error range. In the analysis of geometric tolerance, the parameters of SDT which control geometric features are randomly selected in their value range to obtain the corresponding random sequence. According to the stacking process shown in Figure 8, calculate and repeat this step until the number of repetitions meets the simulation number $N$. The maximum and minimum values of the calculation results are the prediction range of the corresponding assembly accuracy.

\section{Case Study}

In this paper, the assembly of a shaft in the speed reducer is taken as an example for algorithm verification, implemented by Matlab 2017b and Solidworks API programming. The shaft is mainly composed of shaft, key, gear, locating ring, end cover, sleeves, two bearing, gaskets, and screw. In order to save analysis time and cost and improve disassembly efficiency, we use one fastener to express multiple fasteners. A three-dimensional model of a shaft in the speed reducer is shown in Figure 12 and Table 4. The fit relationship between parts in the assembly is shown in Table 5, and the rest fit relationship is clearance fit.

The model is decomposed into Boolean operation sets of several basic subfeatures according to the volume feature decomposition method. The types of planes included in the simple subfeatures are counted, the normal vectors and contact relationships of the planes are determined, and the assembly information model is established. According to the simplified method proposed in this paper, the port features of each part are determined and the importance of neutron characteristics of each part is ranked. The importance of each subfeature of the most complex shaft part is shown in Figure 4(c) and is then sequenced on this basis. Obtain the contact relationship between the features, and establish the undirected diagram of the contact relationship between the features, as shown in Table 6. Parts are gradually simplified by an algorithm until further simplification is not possible. The feasible movement direction of each part under assembly constraints and the geometrically feasible sequence of mechanical products are obtained through the contact relationship between the surfaces of the parts.

According to the results, the simplified shafts are composed of 9 features, and 11 are reduced. It retains all features of contact with other parts; the volume is $48.98 \mathrm{dm}^{3}$ before simplifying, the reduction volume is $37.55 \mathrm{dm}^{3}$, the volume change is $11.86 \mathrm{dm}^{3}$, and the reduction ratio is about $23.3 \%$. It retains the number of surfaces of 46 before simplifying, and the number of surfaces after simplifying is 26 , the number of surfaces reduced is 20 , and the reduction percentage is about $43.5 \%$. The characteristic topology of the simplified model has a change of $24.3 \%$, where the node represents the feature in the part and the line represents the contact relationship between the two features. By simplifying the parts in the assembly and combining the contact relation between the parts, the following assembly relation characteristic diagram is obtained (Figure 13).

Based on the above analysis of geometric constraints between parts in the product, we obtain 36 geometrically feasible assembly sequences as the input of subsequent assembly sequence optimization, which refer to Table 7 for complete information.

Firstly, the disassembly sequence is reversed to obtain the geometrically feasible assembly sequence of the part, and the feasible direction of the part is determined according to the contact relationship of the direct surface of the part. 


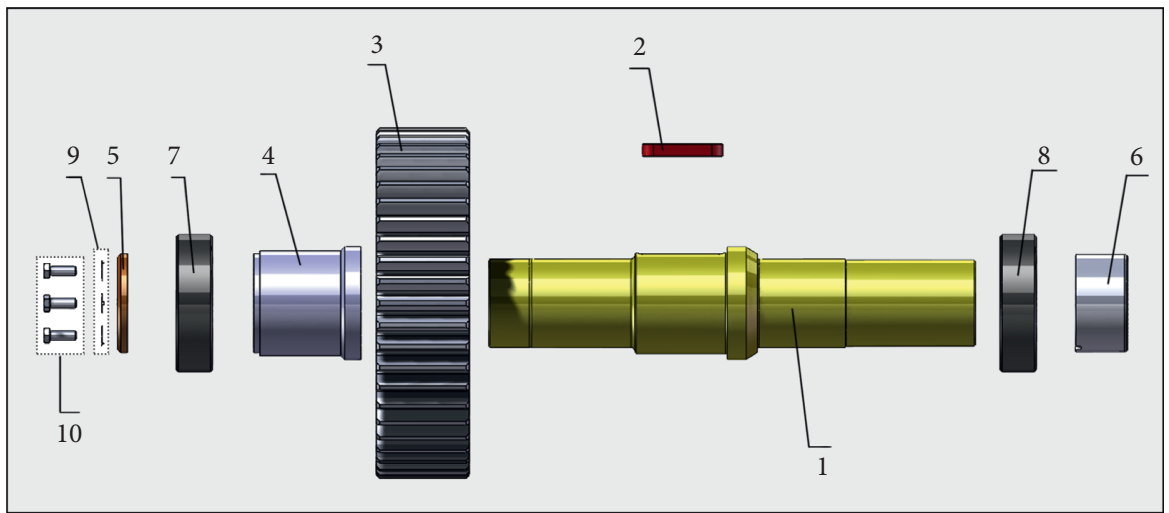

FIGURE 12: Exploded view of reducer shaft and related parts.

TABle 4: The list of features in the shaft.

\begin{tabular}{lccc}
\hline Feature no. & Feature type & Feature volume $v_{i}\left(\mathrm{~mm}^{3}\right)$ & Bool \\
\hline 01 & Step & 13760804 & Port \\
02 & Step & 11191924 & Increase \\
03 & Step & 4618141 & Increase \\
04 & Step & 8286893 & Increase \\
05 & Step & 11625063 & Increase \\
06 & Cone & 202845.4 & Decrease \\
07 & Chamfering & 3081.902 & False \\
08 & Ring channel & 2814.73 & Fecrease \\
09 & Fillet & 15133.4 & Decrease \\
10 & Fillet & 3506.017 & False \\
11 & Fillet & 2716.042 & False \\
12 & Fillet & 2392.43 & False \\
13 & Fillet & 580.5962 & Decrease \\
14 & Chamfering & 3002.734 & Increase \\
15 & Key channel & 221326 & Increase \\
16 & Chamfering & 110.0858 & Decrease \\
17 & Screw hole & False \\
18 & Screw hole & 21714.69 & Decrease \\
19 & Screw hole & 21714.69 & Decrease \\
20 & Screw hole & 21714.69 & False \\
\end{tabular}

TABLE 5: Fit relationship between parts in assembly shown in Figure 11.

\begin{tabular}{lccc}
\hline Reference part no. & Target part no. & Fit type & Value \\
\hline 1 & 3 & Transition & $\mathrm{H} 7 / \mathrm{m} 6$ \\
1 & 7 & Interference & $\mathrm{H} 7 / \mathrm{p} 6$ \\
1 & 8 & Interference & $\mathrm{H} 7 / \mathrm{p} 6$ \\
1 & 10 & Clearance & None \\
\hline
\end{tabular}

According to the sequence, the number of assembly direction changes in different sequences is calculated. The number of tool changes in different sequences is calculated according to the tools required for assembling parts shown in Table 8. Finally, the assembly difficulty of different parts in different sequences is obtained through different matching types. The maximum number of changes of tools is 6 , and the maximum number of direction changes of parts is 5 . The evaluation value of the geometrically feasible assembly sequence is obtained by the adaptability function. The results are shown in Table 9.
TABLE 6: The contact relationship between the features.

$A_{i j}=\left[\begin{array}{llllllllllllllllllll}0 & 1 & 0 & 0 & 0 & 0 & 1 & 1 & 1 & 0 & 0 & 0 & 0 & 0 & 0 & 0 & 1 & 1 & 1 & 1 \\ 1 & 0 & 1 & 0 & 0 & 0 & 0 & 0 & 1 & 1 & 1 & 0 & 0 & 0 & 1 & 1 & 0 & 0 & 0 & 0 \\ 0 & 1 & 0 & 1 & 0 & 1 & 0 & 0 & 0 & 0 & 1 & 1 & 0 & 0 & 0 & 0 & 0 & 0 & 0 & 0 \\ 0 & 0 & 1 & 0 & 1 & 0 & 0 & 0 & 0 & 0 & 0 & 1 & 1 & 0 & 0 & 0 & 0 & 0 & 0 & 0 \\ 0 & 0 & 0 & 1 & 0 & 0 & 0 & 0 & 0 & 0 & 0 & 0 & 1 & 1 & 0 & 0 & 0 & 0 & 0 & 0 \\ 0 & 0 & 1 & 0 & 0 & 0 & 0 & 0 & 0 & 0 & 0 & 0 & 0 & 0 & 0 & 0 & 0 & 0 & 0 & 0 \\ 1 & 0 & 0 & 0 & 0 & 0 & 0 & 0 & 0 & 0 & 0 & 0 & 0 & 0 & 0 & 0 & 0 & 0 & 0 & 0 \\ 1 & 0 & 0 & 0 & 0 & 0 & 0 & 0 & 0 & 0 & 0 & 0 & 0 & 0 & 0 & 0 & 0 & 0 & 0 & 0 \\ 1 & 1 & 0 & 0 & 0 & 0 & 0 & 0 & 0 & 0 & 0 & 0 & 0 & 0 & 0 & 0 & 0 & 0 & 0 & 0 \\ 0 & 1 & 0 & 0 & 0 & 0 & 0 & 0 & 0 & 0 & 0 & 0 & 0 & 0 & 0 & 0 & 0 & 0 & 0 & 0 \\ 0 & 1 & 1 & 0 & 0 & 0 & 0 & 0 & 0 & 0 & 0 & 0 & 0 & 0 & 0 & 0 & 0 & 0 & 0 & 0 \\ 0 & 0 & 1 & 1 & 0 & 0 & 0 & 0 & 0 & 0 & 0 & 0 & 0 & 0 & 0 & 0 & 0 & 0 & 0 & 0 \\ 0 & 0 & 0 & 1 & 1 & 0 & 0 & 0 & 0 & 0 & 0 & 0 & 0 & 0 & 0 & 0 & 0 & 0 & 0 & 0 \\ 0 & 0 & 0 & 0 & 1 & 0 & 0 & 0 & 0 & 0 & 0 & 0 & 0 & 0 & 0 & 0 & 0 & 0 & 0 & 0 \\ 0 & 1 & 0 & 0 & 0 & 0 & 0 & 0 & 0 & 0 & 0 & 0 & 0 & 0 & 0 & 1 & 0 & 0 & 0 & 0 \\ 0 & 1 & 0 & 0 & 0 & 0 & 0 & 0 & 0 & 0 & 0 & 0 & 0 & 0 & 1 & 0 & 0 & 0 & 0 & 0 \\ 1 & 0 & 0 & 0 & 0 & 0 & 0 & 0 & 0 & 0 & 0 & 0 & 0 & 0 & 0 & 0 & 0 & 0 & 0 & 0 \\ 1 & 0 & 0 & 0 & 0 & 0 & 0 & 0 & 0 & 0 & 0 & 0 & 0 & 0 & 0 & 0 & 0 & 0 & 0 & 0 \\ 1 & 0 & 0 & 0 & 0 & 0 & 0 & 0 & 0 & 0 & 0 & 0 & 0 & 0 & 0 & 0 & 0 & 0 & 0 & 0 \\ 1 & 0 & 0 & 0 & 0 & 0 & 0 & 0 & 0 & 0 & 0 & 0 & 0 & 0 & 0 & 0 & 0 & 0 & 0 & 0\end{array}\right]$.



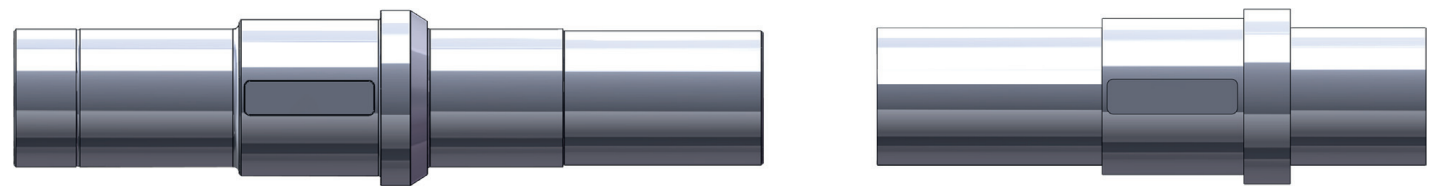

(a)

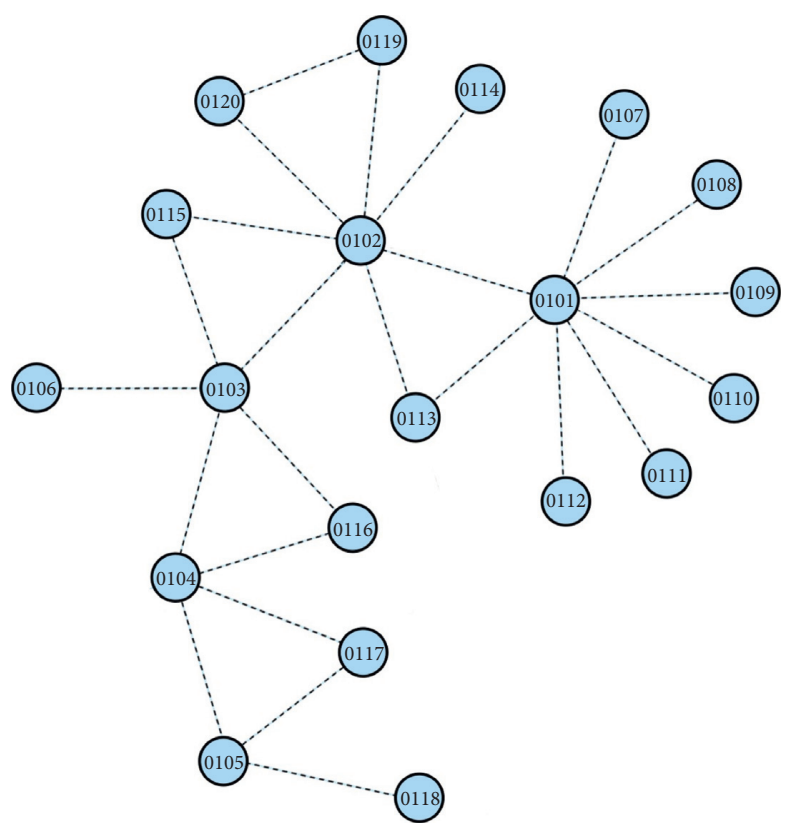

(b)

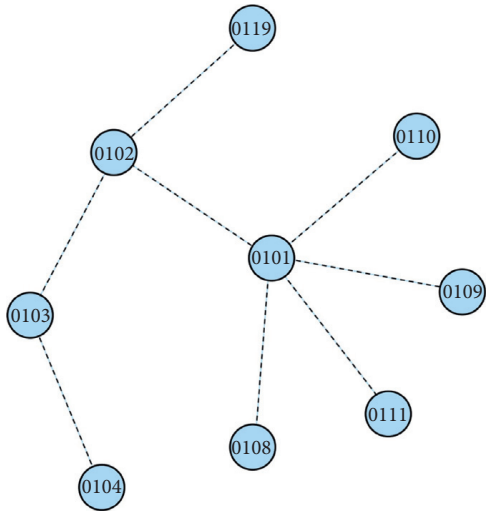

(c)

Figure 13: Part simplification results. (a) Before and after shaft simplification. (b) Topological relations of features before simplification. (c) Topological relations of features after simplification.

Based on the assembly sequence of geometrically feasible parts, the breadth-first algorithm is applied to obtain three optimal sequences: 1-2-3-4-7-5-9-10-8-6, 1-2-8-6-3-4-7-59-10, or 1-8-6-2-3-4-7-5-9-10.

The topological relationship between parts can be represented as the transfer matrix of assembly error based on the surface by the undirected graph. Taking the axis as an example, the simplified shaft surface relationship is shown in Figure 14(a), while the relationship between the axis surface after adding dimension tolerance and form position tolerance is shown in Figure 14(b). The error transfer model of the assembly 
TABLe 7: Assembly sequence planning results.

\begin{tabular}{|c|c|c|c|c|c|c|c|c|c|c|c|c|c|c|c|c|c|}
\hline No. 1 & No. 2 & No. 3 & No. 4 & No. 5 & No. 6 & No. 7 & No. 8 & No. 9 & No. 10 & No. 11 & No. 12 & No. 13 & No. 14 & No. 15 & No. 16 & No. 17 & No. 18 \\
\hline 1 & 1 & 1 & 1 & 1 & 1 & 1 & 1 & 1 & 1 & 1 & 1 & 1 & 1 & 1 & 1 & 1 & 1 \\
\hline 2 & 2 & 2 & 2 & 2 & 2 & 2 & 2 & 2 & 2 & 2 & 2 & 2 & 2 & 2 & 2 & 2 & 2 \\
\hline 3 & 3 & 3 & 3 & 3 & 3 & 3 & 3 & 3 & 3 & 3 & 3 & 3 & 3 & 3 & 3 & 3 & 3 \\
\hline 4 & 4 & 4 & 4 & 4 & 4 & 4 & 4 & 4 & 4 & 4 & 4 & 4 & 4 & 4 & 8 & 8 & 8 \\
\hline 7 & 7 & 7 & 7 & 7 & 7 & 7 & 7 & 7 & 7 & 8 & 8 & 8 & 8 & 8 & 4 & 4 & 4 \\
\hline 5 & 5 & 5 & 5 & 5 & 5 & 8 & 8 & 8 & 8 & 6 & 7 & 7 & 7 & 7 & 6 & 7 & 7 \\
\hline 8 & 8 & 8 & 9 & 9 & 9 & 5 & 5 & 5 & 6 & 7 & 5 & 5 & 5 & 6 & 7 & 5 & 5 \\
\hline 6 & 9 & 9 & 8 & 8 & 10 & 6 & 9 & 9 & 5 & 5 & 6 & 9 & 9 & 5 & 5 & 6 & 9 \\
\hline 9 & 6 & 10 & 6 & 10 & 8 & 9 & 6 & 10 & 9 & 9 & 9 & 6 & 10 & 9 & 9 & 9 & 6 \\
\hline 10 & 10 & 6 & 10 & 6 & 6 & 10 & 10 & 6 & 10 & 10 & 10 & 10 & 6 & 10 & 10 & 10 & 10 \\
\hline No. 19 & No. 20 & No. 21 & No. 22 & No. 23 & No. 24 & No. 25 & No. 26 & No. 27 & No. 28 & No. 29 & No. 30 & No. 31 & No. 32 & No. 33 & No. 34 & No. 35 & No. 36 \\
\hline 1 & 1 & 1 & 1 & 1 & 1 & 1 & 1 & 1 & 1 & 1 & 1 & 1 & 1 & 1 & 1 & 1 & 1 \\
\hline 2 & 2 & 2 & 2 & 2 & 2 & 2 & 2 & 2 & 2 & 8 & 8 & 8 & 8 & 8 & 8 & 8 & 8 \\
\hline 3 & 3 & 3 & 8 & 8 & 8 & 8 & 8 & 8 & 8 & 2 & 2 & 2 & 2 & 2 & 2 & 2 & 6 \\
\hline 8 & 8 & 8 & 3 & 3 & 3 & 3 & 3 & 3 & 6 & 3 & 3 & 3 & 3 & 3 & 3 & 6 & 2 \\
\hline 4 & 4 & 6 & 4 & 4 & 4 & 4 & 4 & 6 & 3 & 4 & 4 & 4 & 4 & 4 & 6 & 3 & 3 \\
\hline 7 & 7 & 4 & 6 & 7 & 7 & 7 & 7 & 4 & 4 & 6 & 7 & 7 & 7 & 7 & 4 & 4 & 4 \\
\hline 5 & 6 & 7 & 7 & 5 & 5 & 5 & 6 & 7 & 7 & 7 & 5 & 5 & 5 & 6 & 7 & 7 & 7 \\
\hline 9 & 5 & 5 & 5 & 6 & 9 & 9 & 5 & 5 & 5 & 5 & 6 & 9 & 9 & 5 & 5 & 5 & 5 \\
\hline 10 & 9 & 9 & 9 & 9 & 6 & 10 & 9 & 9 & 9 & 9 & 9 & 6 & 10 & 9 & 9 & 9 & 9 \\
\hline 6 & 10 & 10 & 10 & 10 & 10 & 6 & 10 & 10 & 10 & 10 & 10 & 10 & 6 & 10 & 10 & 10 & 10 \\
\hline
\end{tabular}

TABLE 8: Tool for the shaft in speed reducer assembly shown in Figure 12.

\begin{tabular}{lcc}
\hline Part no. & Part name & Tool \\
\hline 1 & Shaft & None \\
2 & Key & None \\
3 & Gear & None \\
4 & Locating ring & None \\
5 & End cover & None \\
6 & Sleeves & Tool group \\
7 & Bearing & Tool group \\
8 & Bearing & None \\
9 & Gaskets & Screwdriver \\
10 & Screw
\end{tabular}

TABle 9: Assembly sequence planning results.

\begin{tabular}{lccc}
\hline Assembly sequence & Number of assembly direction changes & Number of assembly tool changes & Fitness function value \\
\hline $1-2-3-8-4-6-7-5-9-10$ & 5 & 5 & 0.867 \\
$1-2-3-4-7-5-9-10-8-6$ & 2 & 5 & 0.667 \\
$1-8-2-3-4-7-5-9-6-10$ & 4 & 5 & 0.867 \\
$1-8-2-3-4-7-5-9-10-6$ & 3 & 6 & 0.800 \\
\hline
\end{tabular}

product is built after the error transfer model of each part is established. According to the algorithm proposed in the previous paper, the error transfer path in the part is obtained, as shown in Figure 15.
Table 10 shows the results of 10,100,1000, and manual dimensional chain calculations. The comparison of data shows that the error obtained by multiple iterations meets the manual calculation results and improves the accuracy. 


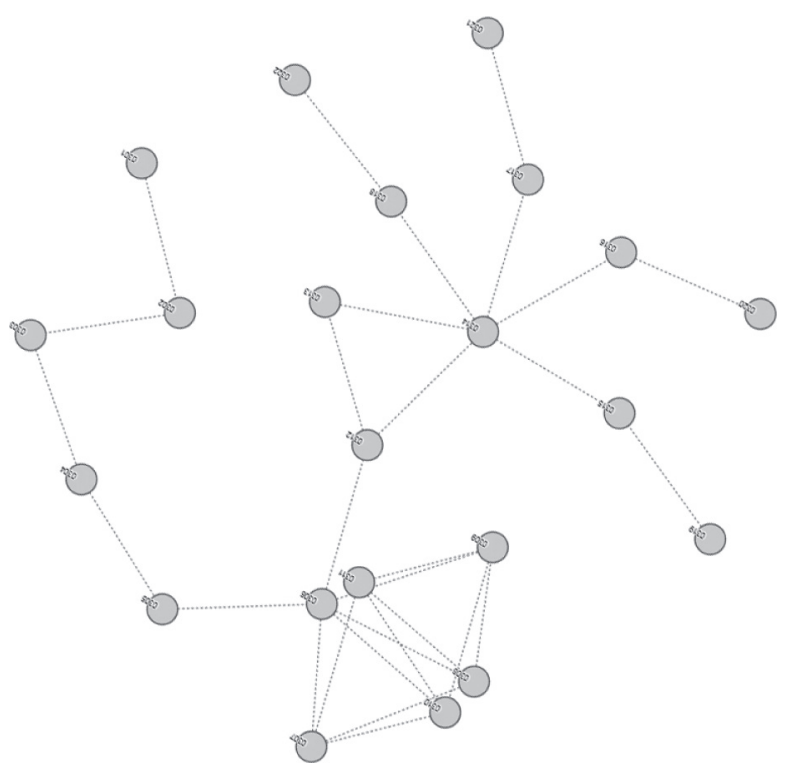

(a)

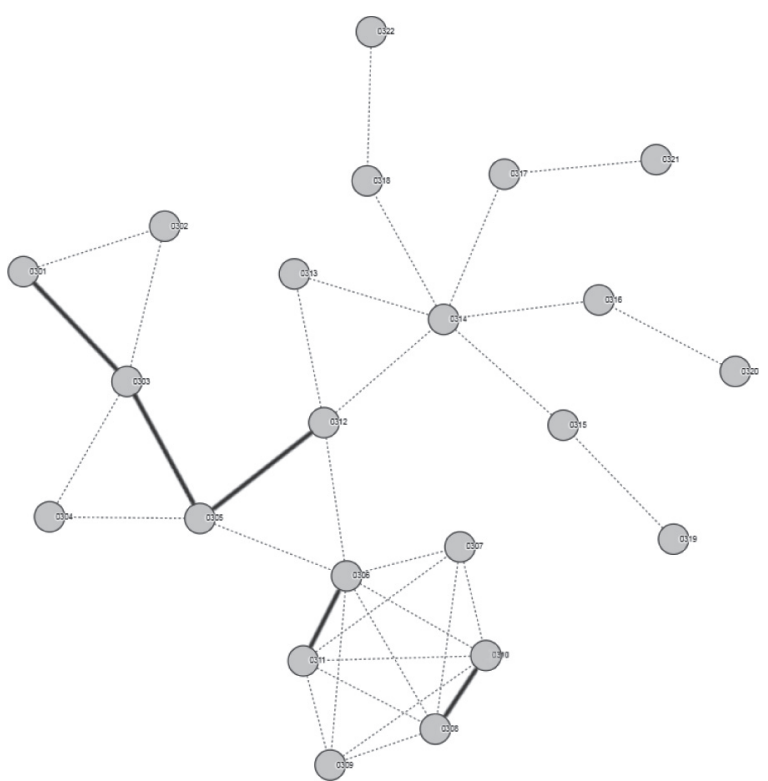

(b)

FiguRE 14: Tolerance transfer model of the shaft. (a) Contact relation of the surface in parts. (b) Tolerance relation of the part surface.

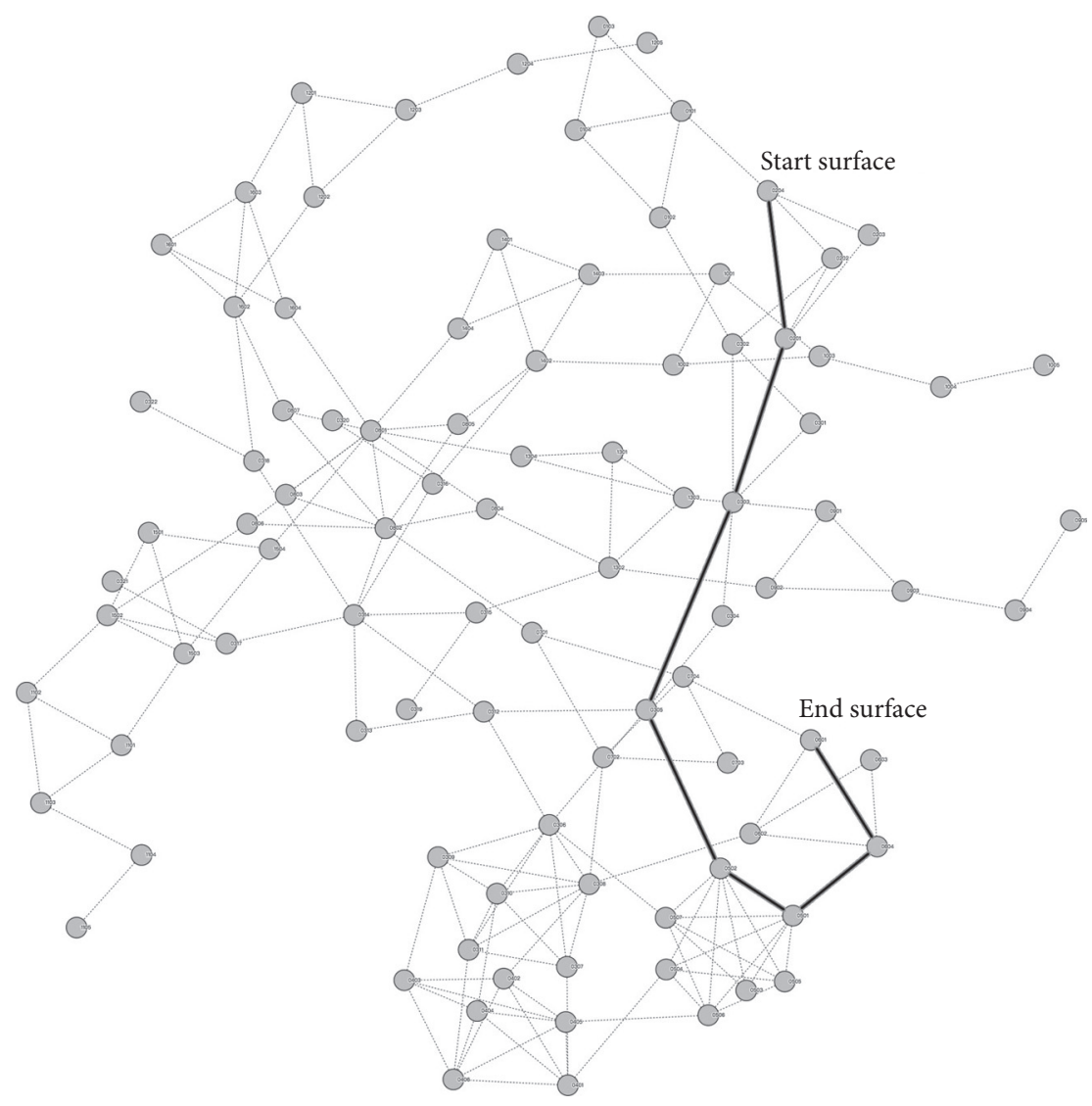

FIgURE 15: Error transmission path. 
TABLE 10: Accuracy calculation results.

\begin{tabular}{lcccc}
\hline & 10 times & 100 times & 1000 times & Dimensional chain \\
\hline$d^{\text {sup }}(\mathrm{mm})$ & 697.5696 & 697.4567 & 697.4403 & 697.4300 \\
$d^{\text {inf }}(\mathrm{mm})$ & 697.8537 & 697.9559 & 698.0630 & 698.0660 \\
\hline
\end{tabular}

\section{Conclusion}

An assembly information model based on the internal structure of parts and the contact relationship among parts is proposed. Firstly, the product is divided into product layer, part layer, feature layer, surface layer, and constraint layer. The assembly information model is built through the topological relationship of features and the contact relationship between parts. On this basis, the assembly constraint model and assembly error transfer model are deduced from the assembly information model. The corresponding relationship between the factors affecting the assembly performance and the total weight is established, and the $3 \mathrm{D}$ dimension chain search algorithm is constructed. The main contributions of this study are as follows.

(1) The assembly information model with multilevel features of the product, part, and assembly process is introduced as a bridge between the product CAD model and the assembly process. High-level semantic concepts of CAD systems and CAD model data of specific products are integrated into a model framework. The assembly information model is built according to the multilevel and multifactor principle, which is the link of CAD and CAPP integration.

(2) According to the assembly information model, the key features in the assembly process are obtained by using the graph theory to simplify the subsequent calculation complexity.

(3) According to the contact relationship between parts, the feasible assembly area of parts is deduced, and the assembly information undirected graph is established. By analyzing the influence factors of the assembly information model, the fitness function of the assembly process is constructed.

(4) The assembly sequence planning and assembly precision calculation are carried out in a model, and the error transfer dimension chain of the assembly is obtained through the sequence planning results. The assembly sequence is taken as the reference factor of precision calculation, which further improves simulation reality. After obtaining the three-dimensional dimension chain of the product, the assembly accuracy of the product can be calculated, including the error transfer and numerical calculation.

\section{Data Availability}

The data used to support the findings of the study are available from the corresponding author upon request.

\section{Conflicts of Interest}

The authors declare that there are no conflicts of interest regarding the publication of this paper.

\section{References}

[1] G. Prabhaharan, P. Asokan, P. Ramesh et al., "Geneticalgorithm-based optimal tolerance allocation using a leastcost model," International Journal of Advanced Manufacturing Technology, vol. 24, no. 9, pp. 647-660, 2004.

[2] B. Schleich and S. Wartzack, "A quantitative comparison of tolerance analysis approaches for rigid mechanical assemblies," Procedia CIRP, vol. 43, pp. 172-177, 2016.

[3] Z. Zhu and L. Qiao, "Analysis and control of assembly precision in different assembly sequences," Procedia CIRP, vol. 27, pp. 117-123, 2015.

[4] Y. B. Cai, Z. Q. Cao, and Z. C. Li, "The research of complex assembly dimensional chain analyses and calculation," Modern Manufacturing Engineering, vol. 4, pp. 81-84, 2008.

[5] J. K. Davidson, A. Mujezinović, and J. J. Shah, "A new mathematical model for geometric tolerances as applied to round faces," Journal of Mechanical Design, vol. 124, no. 4, pp. 609-622, 2002.

[6] S. Y. Ding and X. H. Zheng, "Precision control of rotors assembly based on improved Jacobian-Torsor theory," Acta Aeronautica et Astronautica Sinica, vol. 42, Article ID 424670, 2021.

[7] A. Desrochers and A. Clément, "A dimensioning and tolerancing assistance model for CAD/CAM systems," The International Journal of Advanced Manufacturing Technology, vol. 9, no. 6, pp. 352-361, 1994.

[8] T. Jing, X. Tian, X. Liu, H. Hu, M. Zhang, and B. Li, “A multiple alternative processes-based cost-tolerance optimal model for aircraft assembly," The International Journal of Advanced Manufacturing Technology, vol. 107, 2020.

[9] Y. Wang and D. Tian, "A weighted assembly precedence graph for assembly sequence planning," International Journal of Advanced Manufacturing Technology, vol. 83, no. 1-4, pp. 99-115, 2016.

[10] G. Feiyan, L. Jianhua, Z. Fang, Z. Yunong, W. Zhongqi, and L. Shaozhuo, "Research on the state-of-art, connotation and key implementation technology of assembly process planning with digital twin," Journal of Mechanical Engineering, vol. 55, no. 17, p. 110, 2019.

[11] M. Shpitalni, G. Elber, and E. Lenz, "Automatic assembly of three-dimensional structures via connectivity graphs," CIRP Annals, vol. 38, no. 1, pp. 25-28, 1989.

[12] L. S. H. Mello and A. C. Sanderson, "And/or graph representation of assembly plans," IEEE Transactions on Robotics \& Automation, vol. 6, no. 2, pp. 188-199, 1986.

[13] M. R. Bahubalendruni and B. B. Biswal, "A review on assembly sequence generation and its automation," Journal of Mechanical Engineering Science, vol. 54, pp. 203-210, 2015.

[14] M. V. A. R. Bahubalendruni, B. B. Biswal, M. Kumar, and R. Nayak, "Influence of assembly predicate consideration on optimal assembly sequence generation," Assembly Automation, vol. 35, no. 4, pp. 309-316, 2015.

[15] L. S. H. de Mello and A. C. Sanderson, "A correct and complete algorithm for the generation of mechanical 
assembly sequences," Robotics \& Automation IEEE Transactions on, vol. 7, no. 2, pp. 228-240, 1991.

[16] U. Thomas, M. Barrenscheen, and F. M. Wahl, "Efficient assembly sequence planning using stereographical projections of C-space obstacles," in Proceedings of the IEEE International Symposium on Assembly and Task Planning, Besancon, France, July 2003.

[17] J. D. Wolter, "A combinatorial analysis of enumerative data structures for assembly planning," in Proceedings. 1991 IEEE International Conference on Robotics and Automation IEEE, Sacramento, CA, USA, April 2002.

[18] R. Almgren, "Comparative topological modelling and analysis of assemblies and assembly systems-an aid in computerized assembly planning," in Proceedings of the IEEE International Conference on Robotics \& Automation, San Diego, CA, USA, May 2002.

[19] X. Kou, Y. Cao, Q. Wang, and H. Qiao, "Sub-assembly recognition algorithm and performance analysis in assembly sequence planning," International Journal of Advanced Manufacturing Technology, vol. 107, pp. 1-4, 2020.

[20] K. Oshihara, T. Hisano, and W. Ueda, "Automatic generation of assembly instructions using step," in Proceedings of the 2001 IEEE International Conference on Robotics \& Automation, vol. 3, pp. 21-26, Seoul, Republic of Korea, May 2001.

[21] C. Pan, S. S. F. Smith, and G. C. Smith, "Determining interference between parts in cad step files for automatic assembly planning," Journal of Computing and Information Science in Engineering, vol. 5, no. 1, pp. 56-62, 2005.

[22] J. Liu, "The state-of-the-art, connotation and developing trends of the products assembly technology," Journal of Mechanical Engineering, vol. 54, no. 11, p. 2, 2018.

[23] B. Schleich, N. Anwer, Z. Zhu, L. Qiao, L. Mathieu, and S. Wartzack, "A comparative study on tolerance analysis approaches," in Proceedings International Symposium on Robust Design-ISoRD14, pp. 29-39, Copenhagen, Denmark, August 2014.

[24] K. Jiang, J. Liu, and R. Ning, "Reasoning and verifying specifications of geometric tolerances based on polychromatic sets theory," Computer Integrated Manufacturing Systems, vol. 21, no. 3, pp. 593-605, 2015.

[25] A. Clement and P. Bourdet, "A study of optimal-criteria identification based on the small displacement screw model," CIRP Annuals-Manufacturing Technology, vol. 37, no. 1, pp. 503-506, 1988.

[26] Y. Liu, Z. Wu, and J. Yang, "Mathematical model of size tolerance for plane based on mathematical definition," Chinese Journal of Mechanical Engineering, vol. 37, no. 9, pp. 12-17, 2001.

[27] A. Clément, A. Rivière, P. Serré, and C. Valade, "The ttrss: 13 constraints for dimensioning and tolerancing," Geometric Design Tolerancing Theories Standards \& Applications, vol. 59, pp. 122-131, 1998.

[28] J. K. Davidson and J. J. Shah, "Geometric tolerances: a new application for line geometry and screws," Journal of $\mathrm{Me}$ chanical Engineering Science, vol. 216, no. 1, pp. 95-103, 2002.

[29] H. Xiao and Y. Zhu, "Tolerance conversion of aircraft component intersection axes based on T-map," China Mechanical Engineering, vol. 30, no. 13, pp. 1558-1567, 2019.

[30] J. Ke, J. K. Davidson, J. J. Shah, and J. Liu, "Using tolerancemaps to transfer datum plane from design tolerancing to machining tolerancing," in Proceedings of the ASME International Design Engineering Technical Conferences \& Computers \& Information in Engineering Conference, Portland, OR, USA, August 2013.
[31] W. Liu, L. J. NingRuxin, and K. Jiang, "Precision predicting based on directed deviation graph modeling and $\mathrm{D}-\mathrm{H}$ methodology," Journal of Mechanical Engineering, vol. 48, no. 7, pp. 125-140, 2012.

[32] S. Boccaletti, M. Ivanchenko, V. Latora, A. Pluchino, and A. Rapisarda, "Detecting complex network modularity by dynamical clustering," Physical Review. E, Statistical, Nonlinear, and Soft Matter Physics, vol. 75, no. 4, Article ID 45102, 2007.

[33] S. Boccaletti, V. Latora, and Y. Moreno, "Complex networks: structure and dynamics," Complex Systems and Complexity Science, vol. 424, no. 5, pp. 175-308, 2006.

[34] P. Zhu, J. Yu, and X. Zheng, "Variation propagation networkbased modeling and error tracing in mechanical assembling process," Journal of Zhejiang University(Engineering Science), vol. 53, no. 8, pp. 1582-1593, 2019.

[35] S. Koo and K. Lee, "Wrap-around operation to make multiresolution model of part and assembly," Computers \& Graphics, vol. 26, no. 5, pp. 687-700, 2002.

[36] B. C. Kim and D. Mun, "Feature-based simplification of boundary representation models using sequential iterative volume decomposition," Computers \& Graphics, vol. 38, pp. 570-577, 2014.

[37] Y. Kang, B. C. Kim, D. Mun, and S. Han, "Method to simplify ship outfitting and offshore plant equipment three-dimensional (3-D) computer-aided design (CAD) data for construction of an equipment catalog," Journal of Marine Science and Technology, vol. 19, no. 2, pp. 185-196, 2014.

[38] J. Wang, J. Liu, W. Liu et al., "Modeling technology of tolerance zone in virtual environment," Computer Integrated Manufacturing Systems, vol. 18, no. 12, pp. 231-238, 2012.

[39] H. Xie and L. Zhong, "Assembly sequence evaluation method based on analytical hierarchy process," Computer Systems \& Applications, vol. 21, no. 2, pp. 72-76, 2012.

[40] Z. Wu, "Stimulated tolerances modeling based on small displacement torsors and tolerances analysis," Machinery Design \& Manufacture, vol. 12, pp. 1205-1207, 2010. 